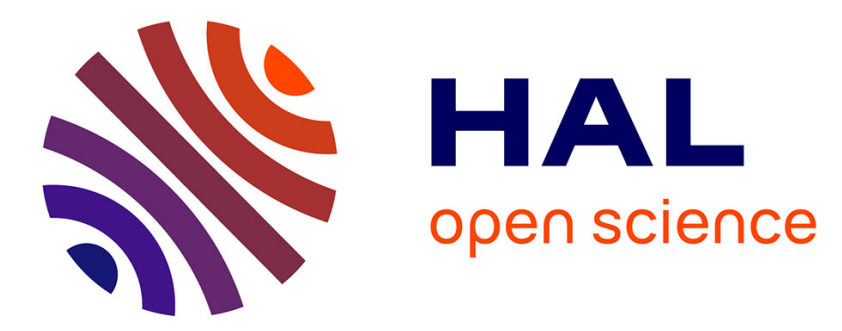

\title{
Interdépendance entre la composition en acides aminés du grain de seigle et son taux d'azote
}

Jacques Baudet, Jean-Claude Huet, Jacques Mossé, Marie-Christine Aubrière, Monique Mansion

\section{- To cite this version:}

Jacques Baudet, Jean-Claude Huet, Jacques Mossé, Marie-Christine Aubrière, Monique Mansion. Interdépendance entre la composition en acides aminés du grain de seigle et son taux d'azote. Agronomie, 1987, 7 (10), pp.813-819. hal-00884957

\section{HAL Id: hal-00884957 \\ https://hal.science/hal-00884957}

Submitted on 1 Jan 1987

HAL is a multi-disciplinary open access archive for the deposit and dissemination of scientific research documents, whether they are published or not. The documents may come from teaching and research institutions in France or abroad, or from public or private research centers.
L'archive ouverte pluridisciplinaire HAL, est destinée au dépôt et à la diffusion de documents scientifiques de niveau recherche, publiés ou non, émanant des établissements d'enseignement et de recherche français ou étrangers, des laboratoires publics ou privés. 


\title{
Interdépendance entre la composition en acides aminés du grain de seigle et son taux d'azote
}

\author{
Jacques BAUDET, Jean-Claude HUET \& Jacques MOSSÉ \\ avec la collaboration technique de Marie-Christine Aubrière et Monique Mansion
}

I.N.R.A., Département de Physiologie et Biochimie végétales, Laboratoire d'Etude des Protéines, Centre de Recherches de Versailles, Route de St-Cyr, F 78000 Versailles

\begin{abstract}
La composition en acides aminés de 13 échantillons de grains de seigle a été déterminée. Ces échantillons ont été choisis parmi 10 génotypes différents et pour leurs taux d'azote (N) qui s'échelonnaient sur une large gamme, de 1,3 à 4,4 p. 100 de la matière sèche. Le taux de chaque acide aminé dans le grain s'avère être une fonction linéaire croissante de $\mathrm{N}$ avec des coefficients de corrélation compris entre 0,98 et 0,99 pour la majorité des acides aminés et inférieurs à 0,965 pour les deux seuls aminés soufrés dont le dosage est moins précis. En conséquence, les teneurs des protéines brutes du grain en chaque acide aminé (en g p. $16 \mathrm{~g}$ d'azote) varient en fonction de $\mathrm{N}$ suivant des fonctions hyperboliques croissantes pour la glutamine plus le glutamate et pour la proline, quasi constantes pour la sérine, la phénylalanine, l'arginine et l'asparagine plus l'aspartate et décroissantes pour tous les autres acides aminés. Ainsi les teneurs en lysine et en tryptophane des protéines des grains les plus pauvres $(\mathrm{N}=1,3)$ sont supérieures de $38 \mathrm{p} .100$ et $56 \mathrm{p}$. 100 , respectivement, à celles des protéines des grains riches en azote $(N=4,4)$. Il en est sensiblement de même pour la glycine, la valine et l'isoleucine (30 à 40 p. 100).

La principale conséquence pratique de ces résultats réside dans le fait que ces relations ne dépendent ni du génotype, ni des conditions agronomiques ou de milieu aboutissant à un phénotype déterminé, de sorte que la seule connaissance du taux $\mathrm{N}$ d'azote du grain de seigle permet d'en déduire sa composition en acides aminés. Les résultats montrent aussi que le coefficient de conversion de l'azote en protéines réelles augmente suffisamment peu avec $\mathrm{N}$ chez le seigle pour être considéré comme constant et de l'ordre de $5,52 \pm 0,3$.
\end{abstract}

Mots clés additionnels : Secale cereale, coefficient de conversion protéine/azote.

The amino-acid composition of 13 samples of rye grains was determined. The samples were selected among 10 different genotypes, for their total nitrogen content $\mathrm{N}$ ranging from 1.3 to $4.4 \%$ of grain dry matter. The level of each amino acid in grain changed as an increasing linear function of $\mathrm{N}$ with correlation coefficients ranging from 0.98 to 0.99 for most of the amino acids and remaining below 0.965 only for sulphur amino acids of which the determination is less accurate. Hence, the amino-acid contents in grain protein (in $\mathrm{g} / 16 \mathrm{~g}$ nitrogen) varied as hyperbolic functions of $\mathrm{N}$. These functions were increasing for proline and glutamine plus glutamate, almost constant for serine, phenylalanine, arginine and asparagine plus aspartate and decreasing for all other amino acids. Thus lysine and tryptophan in rye protein were $38 \%$ and $56 \%$, respectively, higher in low protein grains $(\mathrm{N}=1.3)$ than in high protein grains $(\mathrm{N}=4.4)$. The same was roughly true for glycine, valine and isoleucine which showed the strongest variation $(30$ to $40 \%)$ in the $\mathrm{N}$ range investigated. The main consequence of these results lies in the fact that these relations depend neither on genotype nor on agricultural or environmental factors resulting into a given phenotype. Therefore simply knowing $\mathrm{N}$ enables one to predict the amino-acid composition of any rye grain sample. It was also shown that the nitrogen-to-protein conversion factor increases so little as a function of $\mathrm{N}$ that it can be considered constant and equal to $5.52 \pm 0.3$.

Additional key words : Secale cereale, nitrogen-to-protein conversion factor. 


\section{INTRODUCTION}

Le seigle (Secale cereale L.) tient actuellement une place modeste à l'échelle mondiale : derrière les 4 principales céréales (blé, maïs, riz et orge) dont la production approche, depuis des années, 90 p. 100 du tonnage total des grains de céréales récoltés. Il se place en quatrième et dernière position des céréales secondaires (sorgho, avoine, mil et seigle), avec une production très voisine de celle du mil qui ne représente environ que 1,6 p. 100 du total des céréales (F.A.O., 1986). Pourtant, c'est la seule céréale secondaire dont le tonnage annuel ait légèrement augmenté de 1980 à 1985 . Environ la moitié de ce seigle est produite en Europe où la Pologne, la R.D.A. et la R.F.A. récoltent à elles trois près de 80 p. 100 de la collecte européenne, cependant que l'ensemble de la C.E.E. n'en produit que quelque 20 p. 100. Après une désaffection amorcée vers les années 1960 (BUSHUK, 1976), sa production semble se stabiliser, les accroissements de rendement compensant sensiblement les diminutions de surface de sa culture. En France, son rendement oscille entre 30 et $35 \mathrm{q} / \mathrm{ha}$. Il est moitié moindre à l'échelle mondiale, mais il atteint ou dépasse $50 \mathrm{q} /$ ha dans quelques pays d'Europe (F.A.O., 1986). Aussi n'est-il pas exclu qu'à l'avenir sa production se maintienne au moins à son niveau actuel.

Il s'agit en effet d'une espèce particulièrement robuste et douée de toute une série d'atouts (BUSHUK, 1976) : résistante au milieu salin, elle est relativement apte à la panification (POMERANZ et al., 1984) et possède des caractéristiques nutritionnelles assez favorables par rapport à plusieurs autres céréales. Sa contribution au génome du Triticale en accroît aussi l'intérêt. Cependant, pour des raisons compréhensibles de choix, elle a été l'objet d'une sélection beaucoup moins poussée que les quatre céréales prédominantes et est encore incomplètement connue à divers égards.

A cause de sa parenté avec le blé et l'orge au sein des Triticées, le seigle a fait l'objet de quelques travaux récents d'ordre fondamental qui ont essentiellement porté sur ses prolamines, les sécalines, dont le fractionnement et les propriétés (CHARBONNIER el al., 1981) autant que la structure primaire (AUTRAN et al., 1979) ont permis de dégager des homologies avec les prolamines du blé et de l'orge (BIETZ, 1982 ; KREIS et al., 1985).

Malgré cela, en matière de composition globale en acides aminés des grains, les données de la littérature sont peu nombreuses. En se limitant à la lysine, VILLEGAS et al. (1968 et 1970) ont procédé au criblage de plus de 200 échantillons de provenances très diverses, et même de plusieurs espèces différentes de seigle, échantillons dont les taux d'azote $\mathrm{N}$ s'échelonnaient de 1,15 à 3,56 p. 100 (en $g$ p. $100 \mathrm{~g}$ de matière sèche des grains). Les premières analyses à peu près complètes et faites par voie chimique sur colonnes échangeuses d'ions sont dues à BUSSON et al. (1966), TKACHUK \& IRVINE (1969) et CHEN \& BUSHUK (1970). La première de ces trois compositions a d'ailleurs été prise en compte dans une composition moyenne publiée par la F.A.O. (1970). Il convient d'y ajouter une analyse récente due à PEDERSEN \& EGGUM (1983). Par ailleurs, EPPENDORFER (1975 et 1977) a analysé dix échantillons de la variété Petkus soumise à des fumures différant en nature comme en quantité, obtenant ainsi des grains dont les taux d'azote $\mathrm{N}$ variaient sur une large plage. Cet auteur a eu le mérite de souligner que les divers types de fumure (azotée, potassique, phosphorée) influaient sur la composition en acides aminés des grains seulement de manière indirecte en affectant leur taux d'azote. Il a ainsi montré que, pour une variété donnée, quelles que soient les conditions de fertilisation du seigle, à même teneur en azote $\mathrm{N}$, les grains ont même composition en acides aminés. Il confirmait ainsi les résultats que nous avions interprétés de la sorte chez l'orge (MOSSÉ \& BAUDET, 1969) en concluant que cette correspondance biunivoque entre $\mathrm{N}$ et la composition en acides aminés des grains était valable non seulement au sein d'une même variété, mais aussi au sein de l'ensemble des variétés d'une espèce déterminée.

Cette dernière généralisation a été par la suite confirmée chez des céréales comme le blé (Mosse et al., 1985), le maïs (BAUDET et al., 1986) ou le sorgho (résultats non publiés) aussi bien que chez des légumineuses comme la fève (BAUDET \& MOSSÉ, 1980), le pois (HUET et al., 1987) ou le lupin (MOSSÉ et al., 1987). Tout concourt ainsi à penser qu'il s'agit d'un phénomène général chez les grain(e)s, traduisant en fait une régulation très stricte de l'accumulation des réserves et en particulier de la biosynthèse des protéines de réserve. Aussi le but du présent travail était-il de vérifier si ce type de généralisation s'étendait bien au seigle et, dans l'affirmative, d'en préciser les modalités en raison de leur intérêt pratique et nutritionnel.

\section{MATÉRIEL ET MÉTHODES}

\section{A. Matériel}

Les 13 échantillons de grains de seigle analysés ont été choisis parmi 10 génotypes différents : 9 correspondaient à des lignées provenant de la Station de Génétique et d'Amélioration des Plantes de ClermontFerrand et les 4 autres à des échantillons de la variété Petkus (déjà étudiée par EPPENDORFER (1975) dans une optique similaire) récoltés en 4 lieux et années différents. L'échantillonnage des grains a été effectué à partir de lots de l'ordre de $500 \mathrm{~g}$ qui ont été broyés, après congélation dans l'azote liquide, dans un broyeur à couteaux de type Prometer 14920 pendant $20 \mathrm{~s}$. Après rééquilibrage avec l'atmosphère ambiante, des fractions aliquotes de la farine complète ainsi obtenue ont été prélevées simultanément pour les diverses analyses.

\section{B. Méthodes}

Matière sèche et azote des fractions de farine de seigle ont été déterminés en triple exemplaires respectivement par chauffage à $105^{\circ} \mathrm{C}$ pendant $24 \mathrm{~h}$ et par microkjeldahl avec catalyseur au sélénium. Les acides aminés ont été dosés après $24 \mathrm{~h}$ d'hydrolyse à reflux à $115^{\circ} \mathrm{C}$ de $300 \mathrm{mg}$ de farine dans $300 \mathrm{ml}$ de $\mathrm{HCl} 6 \mathrm{M}$ et passage sur une seule colonne d'un analyseur Kontron Liquimat III couplé à un injecteur automatique K 100 pour les fractions d'hydrolysat et à un intégrateur 
numérique Spectraphysics Autolab. Les conditions de chromatographie sur colonnes échangeuses d'ions de l'analyseur étaient ajustées pour permettre une bonne séparation de tous les acides aminés, à l'exception du tryptophane. Ce dernier a été déterminé séparément sur un hydrolysat barytique de certains échantillons étudiés. Toutes les conditions analytiques utilisées ont déjà été décrites par ailleurs (MOSSÉ et al., 1985 et 1987) avec cette restriction que, dans la présente étude, un seul type d'hydrolysat chlorhydrique a été analysé par échantillon de farine et que l'azote amidé de l'asparagine et de la glutamine n'a pas été dosé.

\section{RESULTATS}

\section{A. Taux d'azote et teneurs en acides aminés des grains et de leurs protéines}

Les taux $\mathbf{N}$ d'azote total des grains étudiés, exprimés en $\mathrm{g}$ d'azote $\mathrm{p} .100 \mathrm{~g}$ de matière sèche des grains varient de 1,29 à 1,98 pour les 4 échantillons de la variété Petkus et de 1,63 à 4,37 pour les 9 lignées (tabl. 1). En ce qui concerne les compositions en acides aminés, il y a lieu de les exprimer sous plusieurs formes nécessaires pour l'interprétation des présents résultats. Elles peuvent être exprimées d'abord en quantité du $i^{\text {ème }}$ acide aminé par rapport à $100 \mathrm{~g}$ de matière sèche du grain : soit en $g\left(A_{i}\right)$ d'acide aminé, soit en $g\left(E_{i}\right)$ de résidu d'acide aminé. Elles peuvent aussi être exprimées en $g\left(C_{i}\right)$ d'acide aminé pour $16 \mathrm{~g}$ d'azote $\mathrm{du}$ grain, expression répandue notamment chez les nutritionnistes. En fait, ce sont les données de la forme $A_{i}$ qui ont été obtenues expérimentalement et à partir desquelles ont été calculées celles du type $C_{i}$ rassemblées dans le tableau 1. Il est cependant éloquent de porter les expressions de la forme $\mathrm{A}_{\mathrm{i}}$ en fonction du taux $\mathrm{N}$ d'azote des grains. C'est ce qui est représenté sur la figure 1, à titre d'exemple, pour la cystéine (exprimée en demi-cystine, suivant l'usage le plus répandu), la lysine et l'arginine. Pour chaque acide aminé i on constate que les points représentatifs des compositions déterminées sont situés au voisinage d'une droite de régression dont l'équation s'écrit :

$$
\mathrm{A}_{\mathrm{i}}=\mathrm{a}_{\mathrm{i}} \mathrm{N}+\mathrm{b}_{\mathrm{i}},
$$

droite de pente $a_{i}$ et d'ordonnée à l'origine $b_{i}$. Les valeurs de ces 2 paramètres et celles des coefficients de régression $r_{i}$ correspondants sont indiquées dans le tableau 2 pour chacun des 18 acides aminés protéiques analysés. Ces coefficients de régression s'avèrent tous supérieurs à 0,95 à l'exception de celui de la cystéine et 14 d'entre eux sont compris entre 0,98 et $0,99,3$ autres seulement étant dans l'intervalle $0,95-0,97$. On est ainsi conduit à conclure que, pour les échantillons de seigle étudiés et aux erreurs analytiques près, la teneur du grain en chaque acide aminé suit une seule et même loi linéaire en fonction de $\mathrm{N}$ quel que soit le génotype parmi les 10 génomes représentés et quel que soit le phénotype qui, par le biais des conditions de culture ou d'environnement, aboutit à un taux $\mathrm{N}$ d'azote déterminé.

En ce qui concerne la teneur $C_{i}$ des protéines totales du grain en l'acide aminé $i$, par définition, elle se déduit de $A_{i}$ par la relation : $C_{i}=16 A_{i} / N$ qui, combinée avec la relation (1) donne :

$$
\mathrm{C}_{\mathrm{i}}=16 \mathrm{a}_{\mathrm{j}}+16 \mathrm{~b}_{\mathrm{j}} / \mathrm{N}
$$

et montre que $\mathrm{C}_{\mathrm{i}}$ est une fonction de $\mathrm{N}$ représentée par un segment d'hyperbole équilatère ayant pour asymptotes l'axe des ordonnées et la droite horizontale d'ordonnée $16 \mathrm{a}_{\mathrm{i}}$.

TABLEAU 1

Composition en acides aminés des échantillons de grain de seigle $\left(\mathrm{g} / 16 \mathrm{~g}\right.$ d'azote $\left.=C_{i}\right)$.

\begin{tabular}{|c|c|c|c|c|c|c|c|c|c|c|c|c|c|}
\hline $\begin{array}{c}\text { Variété (1) } \\
\mathrm{N}\end{array}$ & $\begin{array}{c}P \\
1,29\end{array}$ & $\begin{array}{l}P \\
1,31\end{array}$ & $\begin{array}{l}P \\
1,46\end{array}$ & $\begin{array}{c}1 \\
1,63\end{array}$ & $\begin{array}{c}P \\
1,98\end{array}$ & $\begin{array}{c}2 \\
2,62\end{array}$ & $\begin{array}{c}3 \\
2,80\end{array}$ & $\begin{array}{c}4 \\
3,28\end{array}$ & $\begin{array}{c}5 \\
3,49\end{array}$ & $\begin{array}{c}6 \\
3,55\end{array}$ & $\begin{array}{c}7 \\
3,68\end{array}$ & $\begin{array}{c}8 \\
3,84\end{array}$ & $\begin{array}{c}9 \\
4,37\end{array}$ \\
\hline Gly & 4,56 & 4,71 & 4,33 & 4,56 & 4,19 & 4,06 & 4,43 & 3,55 & 3,55 & 3,91 & 3,75 & 3,55 & 3,50 \\
\hline Ala & 4,53 & 4,71 & 4,15 & 4,61 & 3,94 & 4,21 & 4,15 & 3,85 & 3,80 & 4,16 & 3,80 & 3,60 & 3,70 \\
\hline Val & 4,85 & 5,23 & 4,78 & 5,01 & 4,74 & 4,26 & 4,40 & 4,05 & 3,60 & 4,41 & 3,80 & 3,85 & 3,65 \\
\hline Leu & 6,02 & 6,42 & 5,85 & 6,21 & 5,79 & 5,95 & 5,86 & 5,66 & 5,04 & 5,99 & 5,74 & 5,80 & 5,40 \\
\hline Ile & 3,78 & 3,66 & 3,55 & 3,31 & 3,25 & 3,11 & 3,00 & 3,05 & 2,55 & 3,15 & 2,90 & 3,00 & 2,70 \\
\hline Ser & 4,27 & 4,37 & 4,25 & 4,56 & 4,59 & 4,51 & 4,46 & 4,15 & 3,70 & 4,41 & 4,35 & 4,55 & 3,99 \\
\hline Thr & 3,44 & 3,71 & 3,22 & 3,55 & 3,35 & 3,41 & 3,26 & 2,85 & 2,75 & 3,25 & 3,00 & 3,10 & 2,90 \\
\hline Tyr & 2,53 & 2,86 & 2,36 & 2,86 & 1,95 & 2,60 & 2,50 & 2,45 & 2,10 & 2,40 & 2,55 & 2,55 & 2,10 \\
\hline Phe & 4,02 & 4,42 & 4,15 & 4,86 & 4,64 & 4,55 & 4,70 & 4,40 & 4,05 & 5,00 & 4,80 & 5,15 & 4,54 \\
\hline $\operatorname{Trp}\left({ }^{2}\right)$ & 1,07 & n.d. & 1,00 & n.d. & n.d. & 0,78 & 0,72 & 0,80 & n.d. & n.d. & 0,72 & 0,75 & 0,66 \\
\hline Pro & 7,36 & 8,13 & $8,4 ?$ & 12,98 & 13,27 & 10,06 & 11,80 & 11,51 & 9,60 & 10,41 & 11,52 & 12,60 & 11,99 \\
\hline Met & 1,56 & 1,34 & 1,60 & 1,86 & 1,35 & 1,55 & 1,75 & 1,55 & 1,40 & 1,75 & 1,30 & 1,40 & 1,40 \\
\hline Cys & 2,49 & 0,81 & 2,41 & 2,65 & 2,80 & 2,81 & 1,80 & 1,70 & 1,75 & 2,15 & 1,80 & 2,40 & 1,75 \\
\hline Lys & 4,09 & 4,21 & 3,66 & 4,21 & 3,55 & 3,46 & 3,40 & 3,25 & 3,35 & 3,46 & 3,15 & 3,05 & 2,90 \\
\hline His & 2,07 & 2,26 & 2,05 & 2,21 & 2,09 & 2,00 & 2,00 & 1,95 & 1,70 & 2,06 & 1,90 & 1,80 & 1,70 \\
\hline Arg & 5,20 & 5,52 & 4,87 & 5,26 & 4,64 & 5,16 & 4,50 & 4,76 & 4,91 & 5,21 & 4,75 & 4,60 & 4,85 \\
\hline Asx $\left({ }^{3}\right)$ & 7,89 & 8,28 & 7,07 & 8,51 & 7,03 & 7,27 & 7,89 & 6,95 & 7,24 & 7,75 & 7,04 & 7,04 & 7,54 \\
\hline Glx $\left(^{(3)}\right.$ & 20,71 & 20,76 & 22,90 & 23,08 & 27,31 & 25,53 & 26,06 & 27,27 & 24,11 & 28,21 & 29,83 & 29,25 & 30,76 \\
\hline
\end{tabular}
Amino-acid composition of rye grain samples $\left(\mathrm{g} / 16 \mathrm{~g}\right.$ nitrogen $\left.=C_{i}\right)$.

(1) P: Variété Petkus; 1 à 9 : lignées.

(2) n.d. : non déterminé.

(3) Exprimés respectivement en Asp et Glu. 


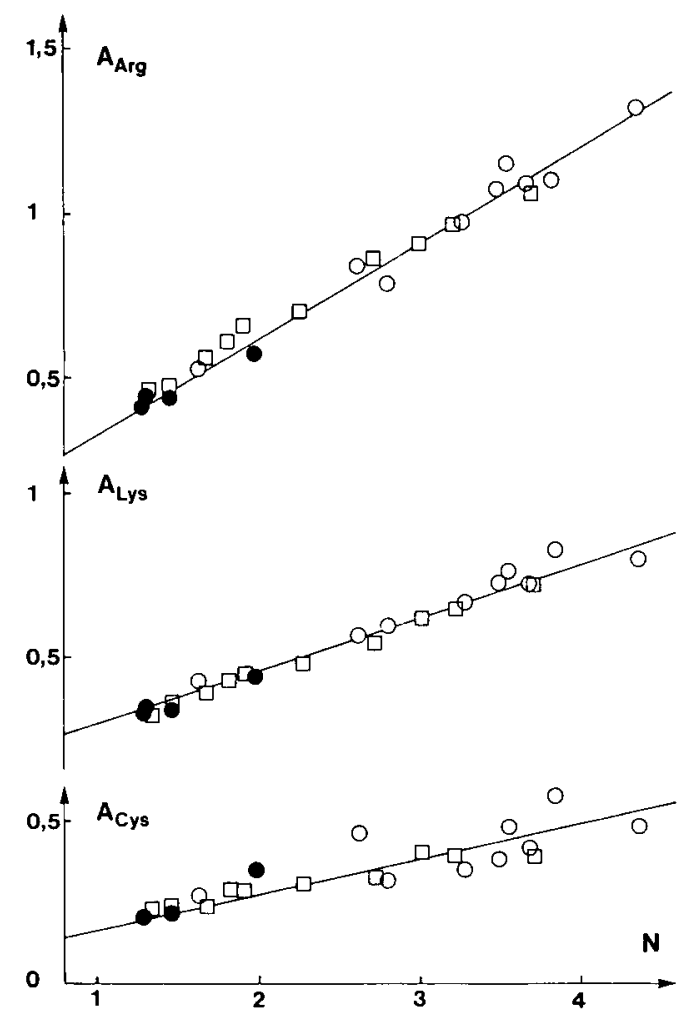

Figure 1

Relations entre la teneur $A_{i} d u$ grain de seigle en cystéine, lysine et arginine et le taux $N$ d'azote du grain. Les disques blancs correspondent à des lignées, les disques noirs à la variété Petkus. Les carrés blancs correspondent aux résultats obtenus également sur la variété Petkus par EPPENDORFER (1975).

Relationships between amino-acid levels $A_{i}$ in rye grain and nitrogen content $N$ for cysteine, lysine and arginine. White circles - rye lines; black circles - variety Petkus; white squares - results fiom EPPENDORFER (1975) also obtained with variety Petkus.

D'un point de vue fondamental, ceci signifie que la teneur en acides aminés des protéines du grain tend vers une teneur limite égale à $16 \mathrm{a}_{\mathrm{i}}$ quand $\mathrm{N}$ augmente. Nous avons déjà eu l'occasion de montrer (MOSSÉ et al., 1986 ; HUET et al., 1987) que cette composition limite n'est autre que celle du supplément de protéines de réserve qui s'accumulent quand on passe de grain(e)s pauvres à des grain(e)s riches en protéines, supplément qui a donc une composition constante.

Selon que le rapport $b_{i} / a_{i}$ est négligeable, négatif ou positif, $C_{i}$ est une fonction quasi constante, croissante ou décroissante de $\mathrm{N}$. Ainsi le rapport $\mathrm{b}_{\mathrm{i}} / \mathrm{a}_{\mathrm{i}}$ est négligeable dans le cas de la sérine, de la phénylalanine, de l'arginine et de l'asparagine plus l'aspartate, acides aminés dont les teneurs dans les protéines varient de moins de 10 p. 100 , en valeur relative, dans l'intervalle de $\mathrm{N}$ en jeu. Ce rapport $b_{i} / a_{i}$ est négatif et non négligeable pour la proline et pour le couple glutamine plus glutamate, lequel augmente de 43 p. 100 quand on passe des protéines de grains à $1,29 \mathrm{p}$. 100 d'azote à celles de grains à 4,37 p. 100 d'azote. Enfin, pour tous les autres acides aminés, le rapport $b_{i} / a_{i}$ est à la fois significatif et positif, si bien que leurs teneurs dans les protéines diminuent quand $\mathrm{N}$ augmente : des grains pauvres en azote $(\mathrm{N}=1,29)$ ont des protéines qui contiennent nettement plus de tryptophane ( 56 p. 100), de valine $(40$ p. 100$)$, de lysine ( 38 p. 100), de glycine (35 p. 100) et d'isoleucine ( 32 p. 100) que celles des
TABLEAU 2

Pente $a_{i}$, ordonnée à l'origine $b_{i}$ et coefficient de corrélation $r_{i}$ des droites de régression entre la teneur en acide aminé $A_{i} d u$ grain et son taux d'azote $N$.

Slope $a_{i}$, intercept $b_{i}$ and correlation coefficient $r_{i}$ of regression lines representing amino-acid level $A_{i}$ in grain against its nitrogen content $N$.

\begin{tabular}{llllll}
\hline & & & & & \\
& $a_{\mathbf{i}} \pm$ é.t. $\left.{ }^{(}\right)$ & & $b_{i} \pm$ é.t. $\left({ }^{(}\right)$ & $\mathbf{r}_{\mathbf{i}}\left({ }^{2}\right)$ \\
\hline Gly & 0,192 & 0,011 & 0,142 & 0,031 & 0,983 \\
Ala & 0,21 & 0,009 & 0,102 & 0,026 & 0,990 \\
Val & 0,197 & 0,013 & 0,173 & 0,036 & 0,979 \\
Leu & 0,330 & 0,014 & 0,079 & 0,042 & 0,990 \\
Ile & 0,152 & 0,009 & 0,102 & 0,026 & 0,982 \\
Ser & 0,253 & 0,014 & 0,040 & 0,041 & 0,983 \\
Thr & 0,167 & 0,009 & 0,078 & 0,026 & 0,984 \\
Tyr & 0,137 & 0,011 & 0,037 & 0,033 & 0,965 \\
Phe & 0,304 & 0,017 & $-0,044$ & 0,050 & 0,983 \\
Trp (3) & 0,033 & 0,002 & 0,044 & 0,007 & 0,982 \\
Pro & 0,785 & 0,062 & $-0,252$ & 0,179 & 0,968 \\
Met & 0,086 & 0,008 & 0,022 & 0,024 & 0,954 \\
Cys & 0,109 & 0,021 & 0,055 & 0,60 & 0,848 \\
Lys & 0,162 & 0,008 & 0,133 & 0,024 & 0,986 \\
His & 0,101 & 0,006 & 0,054 & 0,017 & 0,981 \\
Arg & 0,290 & 0,012 & 0,041 & 0,034 & 0,991 \\
Asx & 0,439 & 0,019 & 0,066 & 0,055 & 0,990 \\
Glx & 2,05 & 0,093 & $-1,003$ & 0,270 & 0,989 \\
\hline \hline
\end{tabular}

(1) é.t. : écart-type.

(2) Niveau de signification pour $r_{i}: 0,68(P=0,01)$, pour tous les acides aminés à l'exclusion du Trp.

(3) Valeurs calculées avec les 8 échantillons du tableau 1 et 2 échantillons de la variété Petkus $(\mathrm{N}=1,81$ et $\mathrm{N}=1,92)$. Niveau de signification pour $r_{i}: 0,77(P=0,01)$.

grains les plus riches en azote $(N=4,37)$, pour ne citer que les acides aminés sujets aux variations les plus importantes dans les protéines.

\section{B. Facteur de conversion de l'azote en protéines}

Pour les grain(e)s, il est possible de définir au moins trois types différents de facteurs de conversion de l'azote en protéines (Mossé et al., 1985). L'un d'entre eux donne, à partir de l'azote total du grain, la masse de protéines réelles, c'est-à-dire la masse des résidus des acides aminés polycondensés dans l'ensemble des chaînes polypeptidiques constituant les protéines du grain, au sens biochimique du terme. Ce coefficient de conversion $\mathrm{k}_{\mathrm{P}}$ peut se déduire de l'expression des teneurs en acides aminés de la forme $\mathrm{E}_{\mathrm{i}}$ (en $\mathrm{g}$ de résidus pour $100 \mathrm{~g}$ de matière sèche du grain). Si en effet $\mathbf{M}_{\mathrm{i}}$ désigne la masse molaire de l'acide aminé $i$, comme un résidu résulte d'un acide aminé par perte d'une molécule d'eau (de masse molaire égale à 18), on a : $E_{i}=A_{i}\left(M_{i}-18\right) / M_{i}$. De ce fait $E_{i}$ est, comme $A_{i}$, une fonction linéaire de $N$. La somme $\Sigma E_{i}$ des valeurs de $E_{i}$ étendue aux 18 acides aminés dosés donne la masse totale des résidus des 20 acides aminés protéiques. Par chance, en effet, glutamate et glutamine (qui, par hydrolyse donne du glutamate) ont la même masse molaire à 1 dalton près, et il en est de même pour le tandem aspartate et asparagine. Par conséquent le quotient $\Sigma \mathrm{E}_{\mathrm{i}} / \mathrm{N}=\mathrm{k}_{\mathrm{P}}$ donne le facteur de conversion 
de l'azote total du grain en protéines réelles. Le calcul donne :

$$
\mathrm{k}_{\mathrm{P}}=5,17-0,14 / \mathrm{N}
$$

et montre que $\mathrm{k}_{\mathrm{p}}$ varie comme une fonction hyperbolique croissante de $\mathrm{N}$, mais dans des limites très modestes : quand $N$ passe de 1,29 à $4,37, k_{P}$ passe de 5,06 à 5,14 , et augmente d'une quantité de l'ordre de 1,6 p. 100 . On peut donc le considérer comme constant et égal à $5,1( \pm 0,04)$. On peut aussi traduire ce résultat par le fait que $100 \mathrm{~g}$ de protéines réelles du grain de seigle correspondent à $100 / \mathrm{k}_{\mathrm{p}}=19,6 \mathrm{~g}$ (et non pas $16 \mathrm{~g}$ ) d'azote du grain.

\section{DISCUSSION}

\section{A. Comparaison des résultats avec ceux de la littéra- ture}

Parmi les trois acides aminés dont les variations avec $\mathrm{N}$ sont représentées sur la figure 1 , deux, à savoir l'arginine et la cystéine, ont été choisis délibérément pour leurs coefficients de régression $r_{i}$ (tabl. 2) qui sont respectivement le plus élevé $(0,991)$ et le plus faible $(0,848)$. Sur cette figure, en plus des présents résultats relatifs aux 13 échantillons analysés, dont 4 correspondent à la variété Petkus, ceux obtenus par EPPENDORFER (1975) sur cette même variété ont aussi été représentés (carrés blancs). En soumettant des seigles de cette variété à des fumures différant en nature (azote, phosphore, potassium) aussi bien qu'en quantité, cet auteur a obtenu des grains dont les taux d'azote s'échelonnent sur une large gamme $(1,33$ à 3,71 p. 100) recouvrant en partie celle des 4 échantillons du présent travail $(1,29$ à 2 p. 100) et la complétant largement vers les hauts taux d'azote. On constate que pour les trois acides aminés représentés sur la figure 1 (comme d'ailleurs pour les autres), les points correspondant aux données d'EPPENDORFER (1975) sont tous au voisinage des droites de régression obtenues dans la présente étude. Autrement dit, les variations de composition en acides aminés dues exclusivement au phénotype de la variété Petkus coïncident avec celles obtenues pour des grains de seigle correspondant à 9 autres génotypes. C'est là un aspect qui a déjà été souligné et discuté à plusieurs autres occasions et sur lequel il n'apparaît pas utile d'insister une nouvelle fois. Aussi bien chez le blé (MOSSÉ et al., 1985), le maïs (BAUDET et al., 1986), le sorgho ou le triticale (résultats non publiés) que chez des légumineuses comme la fève (BAUDET \& Mossé, 1980), le poids (HUET et al., 1987) ou le lupin (MosSÉ et al., 1987), aucun écart significatif n'a été trouvé entre les régressions phénotypiques relatives à une même variété et celles à la fois génotypiques et phénotypiques relatives à un ensemble de génomes différents.

Il apparaît donc fondé de considérer les valeurs des paramètres $a_{i}$ et $b_{i}$ du tableau 2 comme suffisantes pour déduire du taux d'azote $\mathrm{N}$ d'un échantillon de seigle sa composition en acides aminés sans qu'une analyse de ces derniers soit nécessaire. A cet égard, il est intéressant de comparer les quelques composi-
TABLEAU 3

Composition en acides aminés du grain de seigle $\left(C_{i}: \mathrm{g} / 16 \mathrm{~g}\right.$ d'azote) pour deux taux $N$ d'azote différents : comparaison entre les données de la littérature ((1) à (3)) et celles calculables (CC) à partir du présent travail.

Amino-acid composition $C_{i}$ of rye grain proteins ( $\mathrm{g} / 16 \mathrm{~g}$ nitrogen) for two different nitrogen contents $N$ : comparison between data from literature ((1) to (3)) and predicted values (CC).

\begin{tabular}{llllll}
\hline \hline & $(1)$ & $\mathrm{CC}$ & $(2)$ & $(3)$ & $\mathrm{CC}$ \\
\hline N & 1,824 & 1,856 & 1,888 & 2,148 & 2,148 \\
Gly & 4,3 & 4,29 & 4,03 & 4,34 & 4,13 \\
Ala & 4,4 & 4,26 & 3,98 & 4,26 & 4,14 \\
Val & 5,15 & 4,65 & 4,61 & 4,75 & 4,44 \\
Leu & 6,3 & 5,96 & 6,15 & 6,16 & 5,87 \\
Ile & 3,8 & 3,32 & 3,4 & 3,5 & 3,2 \\
Ser & 4,5 & 4,4 & 4,54 & 4,32 & 4,35 \\
Thr & 3,4 & 3,35 & 3,03 & 3,34 & 3,25 \\
Tyr & 2,8 & 2,51 & 2,31 & 1,92 & 2,47 \\
Phe & 4,5 & 4,49 & 4,19 & 4,42 & 4,55 \\
Trp & & & & 0,74 & 0,86 \\
Pro & 8,65 & 10,38 & 10,04 & 9,38 & 10,68 \\
Met & 1,65 & 1,57 & 1,55 & 1,46 & 1,54 \\
Cys & 2,5 & 2,21 & 2,02 & 1,9 & 2,15 \\
Lys & 4,1 & 3,74 & 4,23 & 3,4 & 3,58 \\
His & 2,25 & 2,08 & 2,00 & 2,21 & 2,01 \\
Arg & 5,5 & 5,0 & 4,64 & 4,58 & 4,95 \\
Asx & 7,4 & 7,6 & 6,45 & 7,15 & 7,52 \\
Glx & 21,7 & 24,15 & 23,53 & 24,18 & 25,33 \\
& & & & & \\
\hline \hline
\end{tabular}

(1) BussON et al. (1966).

(2) Pedersen \& Eggum (1983).

(3) F.A.O. (1970).

tions publiées dans la littérature avec celles calculables à partir du tableau 2. C'est ce qui a été fait dans le tableau 3 où sont reportées les compositions de 2 échantillons de taux d'azote très voisins : $N=1,824$ (BUSSON et al., 1966) et $\mathrm{N}=1,888$ (PEDERSEN \& EGGUM, 1983) et la composition calculable (CC) pour un taux d'azote intermédiaire (soit $\mathrm{N}=1,856$ ), ainsi que celle d'un échantillon moyen recalculé par la F.A.O. (1970), correspondant à $\mathrm{N}=2,148$, comparée avec celle calculable pour ce dernier taux d'azote. Pour 10 des 17 acides aminés analysés par les deux premiers groupes d'auteurs, ces compositions encadrent systématiquement celle calculable pour un taux d'azote intermédiaire et très voisin. Si on prend la moyenne arithmétique des compositions dues à ces deux groupes, les écarts relatifs les plus élevés par rapport à la composition calculée n'atteignent \pm 8 à \pm 11 p. 100 que pour 4 acides aminés : isoleucine, proline, lysine et asparagine plus aspartate. Pour la composition compilée par la F.A.O. (1970), une demi-douzaine d'acides aminés atteignent des écarts similaires, dont l'isoleucine et la proline qui, comme pour les compositions des deux premiers groupes d'auteurs, s'écartent de la même manière de la composition calculée, cependant que la tyrosine apparaît très sous-estimée $(-22$ p. 100) dans les données de la F.A.O. (1970).

\section{B. Comparaison entre les grains de seigle et de blé}

D'un point de vue nutritionnel, si on se réfère au profil des acides aminés indispensables défini par la F.A.O. (1973), les protéines du seigle, comparées à cel- 
les du blé (Mossé et al., 1985) sont légèrement déficientes en valine comme celles du blé, un peu plus déficientes en leucine et surtout en isoleucine que celles du blé et avant tout pauvres en tryptophane dès que $\mathrm{N}$ dépasse 1,6. Le seigle est ainsi, avec le maïs, quoique à un degré moindre, la seule céréale carencée en tryptophane, dont les teneurs diminuent de 92 à 73 p. 100 de celles du profil de la F.A.O. (1973) quand $\mathrm{N}$ augmente de 2 à 4 . En regard de ces déficiences qui sont d'ailleurs très peu marquées aux faibles taux d'azote, le seigle possède deux atouts importants. Quoique déficient en thréonine comme l'est aussi le blé, il en est néanmoins un peu mieux pourvu et surtout ses protéines contiennent 25 à $30 \mathrm{p}$. 100 de plus de lysine que celles du blé : pour $\mathrm{N}=2$, elles atteignent $74 \mathrm{p} .100 \mathrm{du}$ niveau standard défini par la F.A.O. (1973) alors que celles du blé n'atteignent que $60 \mathrm{p}$. 100, et pour $\mathrm{N}=4,57$ p. 100 au lieu de 42 p. 100 chez le blé. Audessus de $\mathrm{N}=2$, le seigle est aussi plus riche en lysine que l'orge (MOSSÉ \& BAUDET, 1977), ce qui en fait la céréale la moins déficiente en lysine parmi les Triticées.

A notre connaissance le facteur de conversion $k_{P}$ n'avait pas encore été déterminé chez le seigle. Cependant un facteur voisin $\mathrm{k}_{\mathrm{A}}$ a été déterminé notamment par TKACHUK (1969). Il consiste à rapporter la masse $\Sigma \mathrm{E}_{\mathrm{i}}$ des résidus d'acides aminés, non pas à l'azote total du grain, mais à une grandeur légèrement inférieure, à savoir l'azote des acides aminés (non déterminée dans la présente étude, faute de connaître l'azote amidé de l'asparagine et de la glutamine). Par définition, $\mathrm{k}_{\mathrm{A}}>\mathrm{k}_{\mathrm{P}}$ puisque l'azote total inclut un peu d'azote non protéique en plus de celui des acides aminés. Pour le seigle, TKACHUK (1969) trouve $\mathrm{k}_{\mathrm{A}}=5,64$, valeur effectivement supérieure à celle de $\mathrm{k}_{\mathrm{P}}=5,1$ trouvée dans la présente étude. On peut remarquer aussi que, chez le seigle, $\mathrm{k}_{\mathrm{P}}$ est pratiquement toujours inférieur à celui du blé qui passe de 5,25 pour $\mathrm{N}=2$ à 5,37 pour $\mathrm{N}=3$ (MossÉ et al., 1985). De la sorte, par g d'azote du grain, il y a un peu plus de protéines réelles dans le seigle que dans le blé, mais la différence, de l'ordre de 4 p. 100 en valeur relative, est très modeste.

\section{CONCLUSION}

En bref, on peut retenir que, dans l'intervalle très large des taux d'azote en jeu dans cette étude $(1,3$ à 4,4 p. 100), le niveau de chaque acide aminé du seigle varie en fonction de $\mathrm{N}$ comme chez les autres céréales dans des conditions qui permettent de connaître la composition d'un quelconque échantillon de grains à partir d'une simple détermination de $\mathrm{N}$. De plus, la linéarité entre $A_{i}$ et $N$ permet d'utiliser les présents résultats pour n'importe quel lot de grains de seigle, si hétérogène soit-il : mélange de différentes variétés, mélange de lots à taux d'azote différents. Comme le seigle a des protéines mieux pourvues en lysine que les autres Triticées, c'est là un élément susceptible d'être pris en considération aussi bien en vue de la sélection de seigles riches en protéines que dans l'utilisation de cette espèce en alimentation humaine ou animale.

Reçu le 10 juin 1987. Accepté le 18 août 1987.

\section{REMERCIEMENTS}

Nous sommes redevables à nos collègues de la Station de Génétique et d'Amélioration des Plantes de l'I.N.R.A. de ClermontFerrand ainsi qu'à MM. LeUiLLET et MAUPETIT pour les échantillons de grains qu'ils ont réussi à nous fournir et les en remercions vivement.

\section{RÉFÉRENCES BIBLIOGRAPHIQUES}

Autran J. C., Lew E. J. L., Nimmo C. C., Kasarda D. D., 1979. NTerminal amino acid sequencing of prolamins from wheat and related species. Nature, 282, 527-529.

Baudet J., Mossé J., 1980. Amino acid composition of different cultivars of broad beans (Vicia faba). Comparison with other legume seeds. In D. A. Bond, World Crops: production, utilization and description. Vol. 3, Vicia faba : feeding value, processing and viruses. Martinus Nijhoff Publishers. The Hague, Boston, London for the C.E.C., p. 67-82.

Baudet J., Huet J. C., Mossé J., 1986. Variability and relationships among amino acids and nitrogen in maize grains. J. Agric. Food Chem., 34, 365-370.

Bietz J. A., 1982. Cereal prolamin evolution and homology revealed by sequence analysis. Biochem. Genet., 20, 1039-1053.

Bushuk W., 1976. History, world distribution, production and marketing. In W. Bushuк, Rye : production, chemistry and technology. AACC, Inc., St-Paul (Minn.) U.S.A., p. 1-11.

Busson F., Fauconneau G., Pion R., Montreuil J., 1966. Influence des conditions de production de récolte et de stockage sur la composition biochimique des céréales. Répercussion des variations sur la valeur nutritionnelle. Ann. Nutr. Alim., 20, 199-219.

Charbonnier L., Tercé-Laforgue T., Mossé J., 1981. Rye prolamines : extractability, separation and characterization. J. Agric. Food Chem., 29, 968-973.

Chen C. H., Bushuk W., 1970. Nature of proteins in Triticale and its parental species. I. Solubility characteristics and amino acid composition of endosperm proteins. Can. J. Plant Sci., 50, 9-14.
Eppendorfer W. H., 1975. Effects of fertilizers on quality and nutritional value of grain protein. In "Fertilizer use and protein produc tion." Proc. 11 th Coll. Internat. Potash Inst., Rönne-Bornholm, Dk. IPI Berne, p. 249-263.

Epperdorfer W. H., 1977. Nutritive value of oat and rye grain protein as influenced by nitrogen and amino acid composition. J. Sci. Food Agric., 28, 152-156.

F.A.O., 1970. Teneur des aliments en acides aminés et données biologiques sur les protéines. Etudes de nutrition de la F.A.O. N ${ }^{\circ} 24$.

F.A.O., 1973. Energy and protein requirements. Report of a joint F.A.O./W.H.O. ad hoc expert committee. Etudes de nutrition de la F.A.O. $\mathrm{n}^{\circ}$ 52, F.A.O. Rome.

F.A.O., 1986. Annuaire F.A.O. de la production, Rome, 39.

Huet J. C., Baudet J., Mossé J., 1987. Constancy of composition of storage proteins deposited in Pisum sativum seeds. Phytochemistry, 26, 47-50.

Kreis M., Forde B. G., Rahman S., Miflin B. J., Shewry P. R., 1985. Molecular evolution of the seed storage proteins of barley, rye and wheat. J. Mol. Biol., 183, 499-502.

Mossé J., Baudet J., 1969. Etude intervariétale de la qualité protéique des orges : taux d'azote, composition en acides aminés et richesse en lysine. Ann. Physiol. vég., 11, 51-66.

Mossé J., Baudet J., 1977. Relations entre la composition en acides aminés basiques et la teneur en azote des grains d'orge. Europ. Brewery Conv. Proceed 16th Congress, Amsterdam, 27-34. 
Mossé J., Huet J. C., Baudet J., 1985. The amino acid composition of wheat grain as a function of nitrogen content. J. Cereal Sci., 3, 115-130.

Mossé J., Huet J. C., Baudet J., 1986. Evidence from amino acid analysis for the constancy of composition of storage protein accumulated in maize seed. J. Plant Physiol., 125, 199-205.

Mossé J., Huet J. C., Baudet J., 1987. Relationships between nitrogen, amino acids and storage proteins in Lupinus albus seed. Phytochemistry, 26, 2453-2458.

Pedersen B., Eggum B. O., 1983. The influence of milling on the nutritive value of flour from cereal grains. 1. Rye. Qual. Plant Foods Hum. Nutr., 33, 185-196.
Pomeranz Y., Meyer D., Seibel W., 1984. Wheat, wheat-rye, and rye dough and bread studied by scanning electron microscopy. Cereal Chem., 61, 53-59.

Tkachuk R., 1969. Note on the nitrogen to protein conversion factor for wheat flour. Cereal Chem., 43, 223-225.

Tkachuk R., Irvine G. N., 1969. Amino acid compositions of cereals and oilseed meals. Cereal Chem., 46, 206-217.

Villegas E., McDonald C. E., Gilles K. A., 1968. Variability in the lysine content of wheat, rye and triticale proteins. Res. Bulletin (CIMMYT, Mexico), n ${ }^{\circ}$ 10, p. 1-32.

Villegas E., McDonald C. E., Gilles K. A., 1970. Variability in the lysine content of wheat, rye and triticale proteins. Cereal Chem., 47, 746-757. 